\title{
Exkurs: César Vallejo und die Intellektuellen
}

Erlauben Sie mir an dieser Stelle unserer Vorlesung ein Wort zu einer allgemeinen Problematik, die weit über César Vallejos spezifische Situation in Peru und im damaligen Europa hinausgeht! Es ist Zeit für einen kleinen Exkurs zu Rolle und Funktion des Intellektuellen in unseren westlichen Gesellschaften im 20. und auch noch im 21. Jahrhundert.

Diese gesellschaftliche und politische Rolle wird in César Vallejos späten Gedichten, insbesondere jenen, die unter dem Eindruck der Verhältnisse und Auseinandersetzungen in Spanien standen, überaus deutlich. Vallejo hat wie viele andere Partei ergriffen im Spanischen Bürgerkrieg. Doch ist diese Parteinahme keineswegs eine parteipolitische beziehungsweise zugunsten einer bestimmten politischen Partei, sondern eine Parteinahme für die Partei des Menschen schlechthin. Dies sollte, jenseits aller politischen Optionen, auch das obige Gedicht aus España, aparta de mi este cáliz gezeigt haben. Selbstverständlich besaß Vallejo genauer bestimmbare politische Ansichten und Absichten, die ihn zweifellos spätestens seit seinen Reisen in die Sowjetunion mit den politischen Überzeugungen der Linken und speziell der Kommunisten sympathisieren ließen. Zweifellos zeigte er auch ein politisches Engagement gegen den Faschismus und machte sich von daher für ganz präzise politische Gegen-Optionen und GegenEntwürfe stark. Doch sollten wir seine Rolle - wie auch die Rolle vieler anderer Intellektueller und Künstler seiner Zeit - nicht bloß als parteipolitisch im engeren Sinne oder gar als von einer bestimmten Partei gelenkt deuten.

Wir sollten daher die politische Dimension seines Schreibens wie seines Handelns weiter fassen. Dazu müssen wir freilich innerhalb des damaligen zeithistorischen Kontextes begreifen, dass sich jene Intellektuellen und Künstler verschiedenster Länder Europas und Amerikas, die sich in Spanien zusammenfanden und um die bedrohte Spanische Republik scharten, in eine Tradition einschrieben, die mit einer das gesamte 20. Jahrhundert prägenden politisch-militärischen Urerfahrung begann. Es ist nur wenig übertrieben, wenn wir behaupten, dass diese fundamentale Erfahrung im Grunde bis heute ein dauerhafter Bezugspunkt politischen Handelns intellektueller Gruppen und Individuen geblieben ist. Leitsprüche der spanischen Republikaner - der „rojos“, der „Roten“, wie sie genannt wurden - oder der internationalen Brigaden wie „¡No pasarán!“ sind bis heute geradezu sprichwörtlich geblieben.

Zweifellos kann man von einer Sonderrolle Frankreichs innerhalb dieser langfristigen Entwicklung sprechen. Denn es gibt gleichsam eine spezifisch französische Vorgeschichte der Intellektuellen Frankreichs und allgemeiner des Westens, welche zumindest bis zu Voltaire zurückführt, der sich wiederholt für seine wie auch die Überzeugungen anderer vehement einsetzte - im Übrigen 
auch dann, wenn er diese Überzeugungen selbst nicht gänzlich teilte. Es war Voltaire, der wohl erstmals mit beträchtlicher Breitenwirkung innerhalb der damaligen Gesellschaften die Rolle eines Intellektuellen avant la lettre einnahm. Gewiss: Der Autor des Candide wäre kaum auf die Idee gekommen, sich selbst als einen ,Intellektuellen' zu bezeichnen, denn noch stand der Begriff in diesem uns gebräuchlichen modernen Sinne nicht zur Verfügung. Aber der Sache eines ,Intellektuellen' selbst kam er im Verlauf seiner Karriere als Schriftsteller und Philosoph mit wachsendem Renommee mehrfach sehr nahe.

Voltaires Anliegen war dabei die Absicherung und Stützung universaler Prinzipien und Werte oder, um genauer zu sein, von den französischen „philosophes“ für universalgültig gehaltener Prinzipien, wie sie gegen Ende des 18. Jahrhunderts dann einfließen sollten in die französische und US-amerikanische Verfassung, aber auch in die Erklärung der Menschenrechte, der „Droits de l'homme“. Es ging also nicht um eine Parteinahme für bestimmte Interessengruppen oder Parteien, sondern für die Menschheit insgesamt, verkörpert und repräsentiert in ganz bestimmten Individuen und Gruppen, denen man ihre Rechte vorenthielt. Diese ihrer Rechte, Prinzipien oder Bürgerrechte Beraubten verfügten über keinen Diskurs, hatten keine Stimme, fanden kein Gehör: Sie zählten schlichtweg nicht! Voltaire gab ihnen eine Stimme. Er baute in diesem Zusammenhang, bisweilen gepaart mit seinen geradezu propagandistischen Feldzügen, klare Positionen auf hinsichtlich der Verantwortung von - wie wir heute sagen könnten - Kulturschaffenden, von Menschen also, die in ihrem Bereich, in den Künsten, den Literaturen oder den Wissenschaften, über berufliches Prestige, aber keinerlei politische Macht und Einfluss verfügten. Die Aufgabe dieser Gruppe von Menschen, die sich erstmals mit der europäischen und (hemisphärisch verstanden) amerikanischen Aufklärung abzeichnete, bestand darin, Position nicht für Parteien, sondern für allgemeingültige Prinzipien zu ergreifen und letztere gegen alle Ungerechtigkeit, ja gegen alle Unterdrückung und Gewaltherrschaft, mittels ihres Wortes zu verteidigen.

Der Begriff des „intellectuel“, des Intellektuellen, taucht in Frankreich noch nicht zu Zeiten Voltaires auf, findet sich jedoch erstmals bereits in der ersten Hälfte des 19. Jahrhunderts etwa bei Saint-Simon. Aber beschäftigen wir uns nicht gleich mit der Begriffsgeschichte, sondern fragen einmal aktualistisch, wer oder was denn ein Intellektueller sei und für welche Haltungen und Handlungen er einsteht.

Wenn Sie sich in diesem Saal umgucken, dann sitzen hier - nach landläufiger aktueller Begriffsbestimmung - lauter Intellektuelle. Denn Sie sind alle - und das lässt sich begriffsgeschichtlich in Deutschland bis vor das Dritte Reich zurückverfolgen - Geistesarbeiter, Arbeiter und Arbeiterinnen der Stirne. Ja, so hieß das einmal! Die Abgrenzung scheint also auf den ersten Blick so vollzogen werden 
zu können, dass alle, die sich vorwiegend mit geistigen Dingen beschäftigen, als Intellektuelle bezeichnet werden dürfen. So einfach ist das aber nicht: Denn es gab immer wieder Äußerungen, denen zufolge letztlich alle Menschen Intellektuelle seien, da alle Menschen bestimmte kulturelle und politische Vorstellungen besitzen, Ansichten haben und äußern, politische Ziele verfolgen oder diese wie auch immer unterstützen. Aber macht ein Begriff Sinn, in den alle Menschen eingeschlossen sind?

Nach einer solchen ,Definition‘ wären alle, unabhängig von ihrem jeweiligen Beruf, als Intellektuelle anzusehen. Damit wären die eigentlichen beruflichen Tätigkeiten sekundär, die meinetwegen dominant handwerklich beziehungsweise an körperlicher Arbeit ausgerichtet sein könnten: Auch diese Menschen wären aber keineswegs als Nicht-Intellektuelle auszuschließen. Mit dieser Tatsache ließe sich gut leben, doch hilft sie uns herzlich wenig weiter: Denn wenn alle Intellektuelle sind, dann ist es keiner. Wir müssen uns also auf bestimmte historische Traditionen, Prägungen und Entstehungskontexte bei dieser begrifflichen Frage einlassen, welche sich natürlich auch die Herausgeber eines vor einigen Jahren erschienenen (und selbstverständlich sofort umstrittenen) Handbuchs der französischen Intellektuellen stellen mussten, die Herren Julliard und Winock. ${ }^{1}$

In ihrem Vorwort $\mathrm{zu}$ diesem recht gefahrvollen Unterfangen weisen die beiden französischen Herausgeber auf Jean-Paul Sartres Unterscheidung hin, dass ein Wissenschaftler, der an einer - sagen wir - Atombombe bastelt, noch kein Intellektueller ist. Wohl aber wird er zum Intellektuellen, wenn er ein Manifest verfasst und verteilt, in dem er seine Kollegen wie auch einen breiteren Personenkreis mit den Gefahren und Folgen einer Atombombe konfrontiert und vertraut zu machen versucht. Nicht jeder, der sich in diesem Bereich im Sinne Sartres engagiert, wird aber dadurch schon zum Intellektuellen. Er wird es nur, wenn er über einen gewissen Bekanntheitsgrad verfügt, der zumeist auf einem ganz bestimmten Gebiet erworben wurde, in unserem Falle also bei der Lösung spezifischer physikalischer, mathematischer und nuklearwissenschaftlicher Probleme, die sich beim Bau einer Bombe stellen. Der Intellektuelle oder die Intellektuelle muss daher, das ist eine Vorbedingung, über ein bestimmtes fachspezifisches Prestige oder Renommee verfügen, das auf einem ganz bestimmten Gebiet des Wissens, der Künste oder der Öffentlichkeit erworben wurde.

Diese Notorietät oder Bekanntheit könnten wir mit dem Feldsoziologen Pierre Bourdieu sehr wohl als ein symbolisches Kapital begreifen, das in einem ganz bestimmten Tätigkeitsbereich aufgehäuft wurde, um dann in einem anderen

1 Vgl. Julliard, Jacques / Winock, Michel (Hg.): Dictionnaire des intellectuels français. Les personnes, les lieux, les moments. Paris: Editions du Seuil 1996. 
gesellschaftlichen Bereich - etwa im intellektuellen oder im politischen Feld von der oder dem Intellektuellen investiert zu werden. So nutzt etwa der Wissenschaftler oder die Wissenschaftlerin die wissenschaftliche Reputation als Legitimation des eigenen Auftretens in einem anderen Feld, insbesondere dem der Öffentlichkeit, um dergestalt auf politische Meinungen und Entscheidungen (entscheidenden) Einfluss zu nehmen. Sie können dies in diesen Monaten sehr schön auf einem kleinen, aber gesamtgesellschaftlich nicht unbedeutenden Teil-Feld, dem der Virologie, klar erkennen, insofern Virologen in Zeiten der Corona-Krise mittlerweile nicht mehr nur zu ihren Spezialkenntnissen Stellung beziehen.

Aber die Dinge sind nicht immer einfach zu entscheiden, eine genaue Beurteilung fällt oft schwer. Wie wäre etwa eine vor Jahren veröffentlichte Stellungnahme von dreißig Angehörigen dieser Fakultät zu werten, die gegen Stellenabbaupläne der Regierung protestierten? Von den ursprünglich dreißig Unterzeichnern wurden nur einige Namen in der lokalen Zeitung abgedruckt und das auch wohl nach einem Schlüssel, der gewiss ein wenig seltsam anmuten darf; soweit - seien wir ehrlich - ist es also mit der Bekanntheit zum einen nicht her, sie scheint die engsten Fachkreise im Raum Brandenburg-Berlin nicht zu übersteigen!

Zum zweiten beschäftigt sich dieser offene Brief der dreißig Professorinnen und Professoren im Grunde und im Wesentlichen bloß mit der eigenen Situation. Er beklagt - um nur einige Beispiele zu nennen - natürlich völlig zu Recht, dass die Streichung von Lehrstühlen oder die geplante ,Rückführung' der Mittelbaustellen auf knapp zwei Drittel oder auch die weiterhin prekäre Situation der Kunstgeschichte an dieser Fakultät schlicht ein Unding sei und diese Universität dadurch Gefahr laufe, im Zustand eines Torsos zu verharren. Das ist alles richtig; eine im breiten Sinne gesellschaftliche Reperkussion aber bedeutet dies nicht und ist zugleich argumentativ völlig auf den eigenen Arbeits- und Tätigkeitsbereich abgestellt. Das ist wohlgemerkt legitim und gewiss auch notwendig; doch zu einem Intellektuellen wird man dadurch nicht: zumindest nicht im Sinne von Julliard und Winock (und selbstverständlich auch nicht in meinem Sinne). Selbst wenn man sich eines Mittels und eines Mediums bedient, dass für die Intellektuellen geradezu klassisch geworden ist: eines Offenen Briefes in der Zeitung. Sie sehen, wir beginnen, uns der Frage des Manifests, die im Kontext der Avantgarden ja eine so große Rolle spielt, von einer ganz anderen Seite zu nähern. Womit ich freilich nicht sagen will, dass alle Unterzeichner von Manifesten automatisch Intellektuelle wären: weit gefehlt.

Fahren wir also in unserer Bestimmung der Rolle der Intellektuellen fort! Intellektuelle in einem engeren Wortsinn sind jene Vertreter^innen bestimmter literarischer, wissenschaftlicher, künstlerischer oder publizistischer Bereiche, die sich auf ihrem Gebiet eine Kompetenz und ein Prestige angeeignet haben, welche sie dazu berechtigen, ihre Analyse der gesamten Gesellschaft vorzutragen. Diese 
Intellektuellen im engeren Wortsinne werden so im öffentlichen Leben anderen Bedingungen der Kommunikation und der Öffentlichkeit ausgesetzt, als sie dies in ihrem ursprünglichen oder eigentlichen Feld gewohnt waren. Sie setzen sich somit anderen Normen und Formen der Kommunikation und des VerstandenWerdens aus.

Damit ist zugleich gesagt, dass Politiker dank einer solchen Definition aus dem Bereich der Intellektuellen ausscheiden, da sie sich mit öffentlichen und im engeren Sinne politischen Fragestellungen berufsmäßig, also professionell, auseinandersetzen. Daher sind gemäß unserer Definition etwa der Kultursenator von Berlin, die Beauftragte der Bundesrepublik Deutschland für Kultur und Medien oder auch der Oberbürgermeister von Potsdam keine Intellektuellen, auch wenn man ihnen allen sicherlich intellektuelle Fähigkeiten und Tätigkeiten nicht absprechen kann und sie sich darüber hinaus auch in Bereiche des öffentlichen intellektuellen Lebens gestaltend einbringen. Aber sie mischen sich sozusagen nicht ein, da sie schon von Beginn an mit dabei sind und dafür letztlich auch bezahlt werden. Die Intellektuellen aber mischen sich ein in das, wofür sie eigentlich nicht bezahlt werden, transgredieren also die Grenzen ihrer Tätigkeit, während Politiker^innen dafür da sind, sich um öffentliche Belange professionell zu kümmern.

Die oder der Intellektuelle ist, so paradox dies klingen mag, gemäß einer nachvollziehbaren historischen Entwicklung zur Spezialistin oder zum Spezialisten nicht nur eines jeweiligen Fachgebietes oder Arbeitsfeldes, sondern eben und gerade für das Allgemeine geworden, das nach bestimmten Prinzipien und allgemeinen Normen beurteilt und verteidigt werden muss - und zwar gerade nicht nach parteipolitischem Dafürhalten. Sie sind es, die als Intellektuelle wie Joker plötzlich in allen möglichen Zusammenhängen auftauchen, dabei aber Partei ergreifen nicht etwa für bestimmte politische Parteien, sondern für die Partei der universalgültigen menschlichen Werte. Dies zumindest ist jene Klausel, die schon Julien Benda ${ }^{2}$ den Intellektuellen nachdrücklich ins Stammbuch schrieb.

Dieser Ansicht könnte man eine ganze Menge an Argumenten entgegenhalten: Treiben wir nun an dieser Stelle kurz etwas Begriffs-Geschichte! Historisch unter der Bezeichnung aufgetreten sind die Intellektuellen ja erstmals in Frankreich am Ende des 19. Jahrhunderts, vor mehr als hundert Jahren, als dort der Streit um Schuld oder Unschuld des jüdischen Militärangehörigen, des „Capitaine“ Alfred Dreyfus, entbrannt war. Letzterem hatte man - wie sich nach langen Jahren zähen Ringens herausstellte - die Schuld für militärische Schlamperei und Spionage

2 Vgl. die deutschsprachige Ausgabe von Benda, Julien: Der Verrat der Intellektuellen (La trahison des clercs). Mit einem Vorwort von Jean Améry. Aus dem Französischen von Arthur Merin. Frankfurt am Main - Berlin: Ullstein 1983. 
ganz bewusst aus antisemitischen Gründen in die Schuhe geschoben hatte. Die sogenannte Dreyfus-Affäre ${ }^{3}$ spaltete Frankreich in zwei Lager, die sich unversöhnlich gegenüberstanden. Die Spitzen des französischen Staates bis hinauf zum Präsidenten waren involviert, und die Staatsräson schien ebenso unerbittlich wie unerschütterlich zu verlangen, dass man den unehrenhaft entlassenen Dreyfus weit weg von Frankreich auf die Ile du Diable, auf die Teufelsinsel vor Cayenne, verbringen musste. Das antisemitische Staatskomplott war, so schien es lange, unentwirrbar.

Bis Emile Zola kam! Denn erst Zola, der sich mit seinem fast ausschließlich nur auf Indizien aufruhenden Manifest J'accuse am 13. Januar 1898 an die breite Öffentlichkeit wandte und geschickt die Massenmedien beziehungsweise Printmedien für seine Sache nutzte, brachte die ganze Angelegenheit wieder ins Rollen. Frankreich zerfiel nun endgültig in zwei Lager. Bald schon stellte man den erfolgreichen Romancier vor Gericht, was dieser hatte provozieren wollen; und es dauerte nicht lange, bis man auch gegen den französischen Staatspräsidenten zu ermitteln begann.

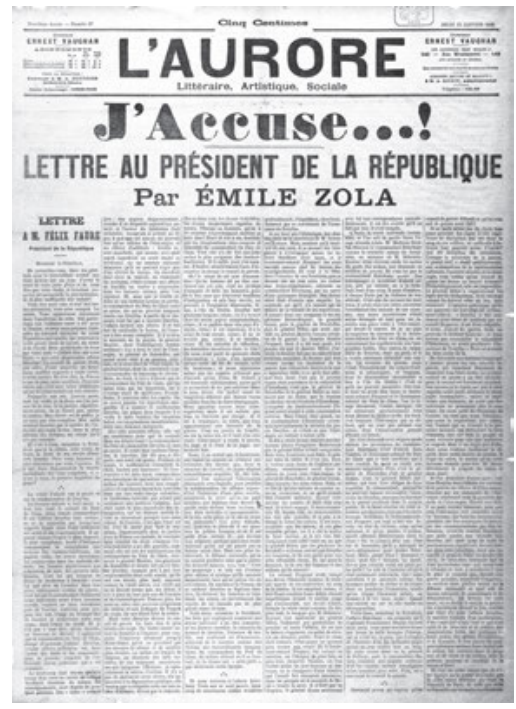

Abb. 65: Titelseite der Zeitung L'Aurore vom 13. Januar 1898 mit Emile Zolas Manifest J'accuse ...!

3 Vgl. u. a. Jurt, Joseph: Die Tradition der europäischen Intellektuellen in Frankreich. Von der Dreyfus-Affaire bis heute. In ders. (Hg.): Intellektuelle - Elite - Führungskräfte. Bildungswege in Deutschland und Frankreich. Freiburg: Frankreich-Zentrum 2004, S. 33-58; ders.: Au nom de la vérité et de la justice. Les valeurs littéraires dans le champ politique au moment de l'Affaire Dreyfus. In: Baudelle, Yves / Deguy, Jacques / Morzevski, Christian (Hg.): A la recherche d'un sens. Littérature et vérité. Mélanges offerts à Monique Gosselin-Noat. Lille: Presses Universitaires du Septentrion 2014, S. 347-359. 
In einem Offenen Brief, dem sogenannten „Protest der Intellektuellen“ - und da haben wir das Wort -, wandten sich viele Schriftsteller, Künstler, Publizisten und Hochschullehrer an die breite französische Öffentlichkeit und forderten mit Emile Zola eine Aufklärung der gegen Alfred Dreyfus erhobenen Vorwürfe, welche zu dessen harter Verurteilung und Verbannung auf die Teufelsinsel geführt hatten. Genauere Analysen der „Affaire Dreyfus“ betonten die Tatsache, dass in diesem öffentlichen Manifest die Unterzeichner häufig ihre Ämter und Funktionen nannten und auch die akademischen Titel mitabgedruckt wurden. Auf diese Weise manifestierte sich jene Funktionsweise, welche gleichsam normbilden innerhalb der französischen Gesellschaft wirkte.

Diese Funktionsweise haben wir bereits erkannt: Die auf einem anderen beruflichen Gebiet erworbene Notorietät oder öffentliche Bekanntheit wurde von den Unterzeichnern umgemünzt in eine bestimmte Legitimität, im öffentlichen Raum für abstrakte Ideen - in diesem Falle für Wahrheit, Gerechtigkeit, Antirassismus und gegen den in Frankreich grassierenden Antisemitismus - eintreten zu können. Oft wurde freilich gerne übersehen, dass auch die Gegenseite bald mobil machte und Gegenerklärungen veröffentlichte, an denen sich wiederum andere Intellektuelle beteiligten, welche ihrerseits für die Werte des Nationalstaats und der höheren Staatsräson Frankreichs eintraten, die über den individuellen Rechten zu stehen habe. Doch lassen Sie uns an dieser Stelle nicht zu sehr in unnütze Details verwickeln!

Der Ausgang der Dreyfus-Affäre ist bekannt: Sie verhalf zwar dem Individuum Alfred Dreyfus kaum mehr zurück in ein wohlgeordnetes bürgerliches Leben, aus dem ihn die Affäre gerissen hatte, sorgte aber dafür, dass antisemitische Tendenzen innerhalb staatlicher Organe und Institutionen breit bekämpft und öffentlich gemacht sowie angeprangert werden konnten. Dies dämmte zwar den in Frankreich bis heute existierenden Antisemitismus nur notdürftig ein, bildete aber einen großen Unterschied zur Situation im Deutschen Reich, welche später, unter den Nationalsozialisten, noch furchtbare Folgen zeitigen sollte. Gewiss: Der Erfolg Zolas und der Intellektuellen in der Dreyfus-Affäre verhinderte nicht, dass zum damaligen Zeitpunkt eine breite Welle des Antisemitismus das Land durchlief und erstmals in neuerer Zeit den Gegensatz zwischen französischer Nation und einer bestimmten Minderheit aufbrechen ließ. Damit wurde zugleich die französische Definition des Nationenbegriffs für längere Zeit in Frage gestellt die Folgen der Affäre waren folglich nicht nur positiv.

Doch insgesamt überwiegen unverkennbar die Durchsetzung rechtsstaatlicher Prinzipien, die Bekräftigung von Wahrheit und Anstand sowie eine ethische Überprüfung staatlicher Grundlagen, wie man sie sich heute etwa für die USA unter Donald Trump - freilich ohne Hoffnung auf jeglichen Erfolg - nur wünschen würde. Doch in den USA - und dies macht durchaus einen grundlegenden 
Unterschied aus - hat es eine mit Frankreich vergleichbare Tradition der Intellektuellen im Sinne einer kritischen, unabhängigen Überprüfung staatlichen Handelns nie gegeben. Vergleichbar mit Diktaturen und autoritären Systemen werden dort, in der sogenannten Führungsmacht der westlichen Wertegemeinschaft, Whistleblowers und andere missliebige Personen wie Julian Assange oder Edward Snowdon, die unbestreitbar grässliche Verbrechen US-amerikanischer Institutionen und Organe aufdecken, im Sinne einer dominanten Staatsräson zu langjährigen Haftstrafen verurteilt. Wahrheit, Justiz, Recht und Anstand zählen angesichts der „raison d'état“ in den Vereinigten Staaten nicht viel. Die Staatsgewalt setzt sich auf eben jene Weise durch, wie sich in Frankreich die antisemitischen Parteigänger der Staatsinteressen durchgesetzt hätten, wäre nicht ein Zola und wären in der Folge nicht zahlreiche „intellectuels“ auf den Plan getreten und hätten die öffentliche Meinung grundlegend verändert.

Wir könnten an dieser Stelle unserer Überlegungen ausführlich über die Analyse von Julliard und Winock in ihrem Dictionnaire des intellectuels français sprechen. Ihr zufolge sei mit der Politisierung der Intellektuellen durch Stellungnahmen für die Sowjetunion in den dreißiger Jahren eine Pervertierung der Rolle und Funktion der Intellektuellen eingetreten, welche Jean-Paul Sartre dann auf die Spitze getrieben habe und die erst dann von einer parteipolitischen Orientierung der Intellektuellen erlöst worden sei, als es mit dem Kommunismus bergab ging. Gerade hier wäre auch die Rolle von César Vallejo mit Blick auf die Sowjetunion zu evaluieren oder auch die Funktion etwa ostdeutscher Intellektueller $\mathrm{zu}$ diskutieren, eine spannende Problematik, welche uns freilich von unserem Thema sehr weit wegführen würde. Mein Ziel war es nur, sie alle ausgehend von unserer Beschäftigung mit den Aktivitäten des späten César Vallejo für derlei Fragestellungen zu sensibilisieren, welche im Grunde die modernen Staatswesen im 20. sowie im 21. Jahrhundert auf ganz grundlegende Weise betreffen. Die „Affaire Dreyfus“ ist daher noch immer ein wesentliches Erbstück unserer Zeit.

Noch ein letztes Wort zu den Intellektuellen: Denn wir müssen andererseits miteinbeziehen, dass etwa im Sinne Antonio Gramscis der organische Intellektuelle sich direkt aus seiner Klasse rekrutiert und zum Teil auch im Gegensatz steht zu den traditionellen Intellektuellen wie den Angehörigen von Wissenschaft und Literatur, von Klerus, Justiz oder auch (frei nach dem Italiener Gramsci) Lehrerschaft. Damit wäre natürlich ein übergeordneter, überparteilicher Standpunkt wieder aufgegeben und gruppenspezifische Interessen und Erfordernisse in den Mittelpunkt des Handelns eines „organischen Intellektuellen“ gerückt.

Doch wie dem auch sei: Mir scheint an dieser Stelle unserer Überlegungen entscheidend, dass das Eingreifen des Intellektuellen in die gesellschaftliche Öffentlichkeit und die Entwicklung des Gemeinwesens ausgehend von den Erfahrungen der „Affaire Dreyfus“ zu einem Handlungsmodell gerade im Bereich 
von Künstlern, Schriftstellern und Publizisten wurde. Dieses führte im Kontext der Bedrohung der Spanischen Republik durch den franquistischen Faschismus zu einer breiten Welle internationaler Unterstützung keineswegs nur aus parteipolitischen Erwägungen heraus. Intellektuelle vieler Länder sahen - das zeigten auch die Antifaschistischen Künstlerkongresse von Valencia und Madrid - im Angriff auf die Spanische Republik Grundfragen und Grundrechte innerhalb eines Europa tangiert, in welchem totalitäre Systeme scheinbar unaufhaltsam auf dem Vormarsch waren. Gegen diesen Vormarsch sollte im Namen von Freiheit und Gerechtigkeit der Widerstand möglichst vieler Individuen organisiert werden. Das traurige Ende dieser Geschichte ist bekannt ...

Wenn wir aus dieser Perspektive das Gedicht Masa von César Vallejo lesen, dann wird deutlich, welche Grundwerte der Solidarität, der Menschlichkeit jenseits parteipolitischer Überzeugungen und Handlungsmuster den peruanischen Intellektuellen César Vallejo bewogen haben dürften, sich vehement für die dort utopisch-konkret skizzierte Einheit des Menschengeschlechts gerade in der Stunde seiner höchsten Entzweiung einzusetzen. Vallejo nahm eine historisch möglich und fast notwendig gewordene Rolle ein, wobei sein ganzes symbolisches Kapital, das er einbringen konnte, seine schriftstellerische Tätigkeit war. Und diese zählte, war Vallejos Prestige als Dichter auch zu Lebzeiten noch recht gering.

César Vallejo kann für uns daher - jenseits aller Ästhetik des Bruchs - als jene Verkörperung einer politisch-künstlerisch-ästhetischen Avantgarde gelten, die außerhalb literarischer Trends, Moden und Bewegungen in unglaublicher Radikalität neue Ausdrucksmöglichkeiten menschlichen Schreibens im Kontext sinnentleerter, absurder Welten suchte und versuchte. Lassen Sie uns nun aber zu einer weiteren Figur der Avantgarde kommen, die neben Vicente Huidobro und César Vallejo für eine dritte mögliche Variante nicht nur in der spanischsprachigen Welt stand! 